\title{
CMEARTICLE
}

\section{Clinics in diagnostic imaging (188)}

Mark Christopher Pearce ${ }^{1}$, MD, Garry Choy $^{2}$, MD, Robert Chun Chen $^{3}$, MD
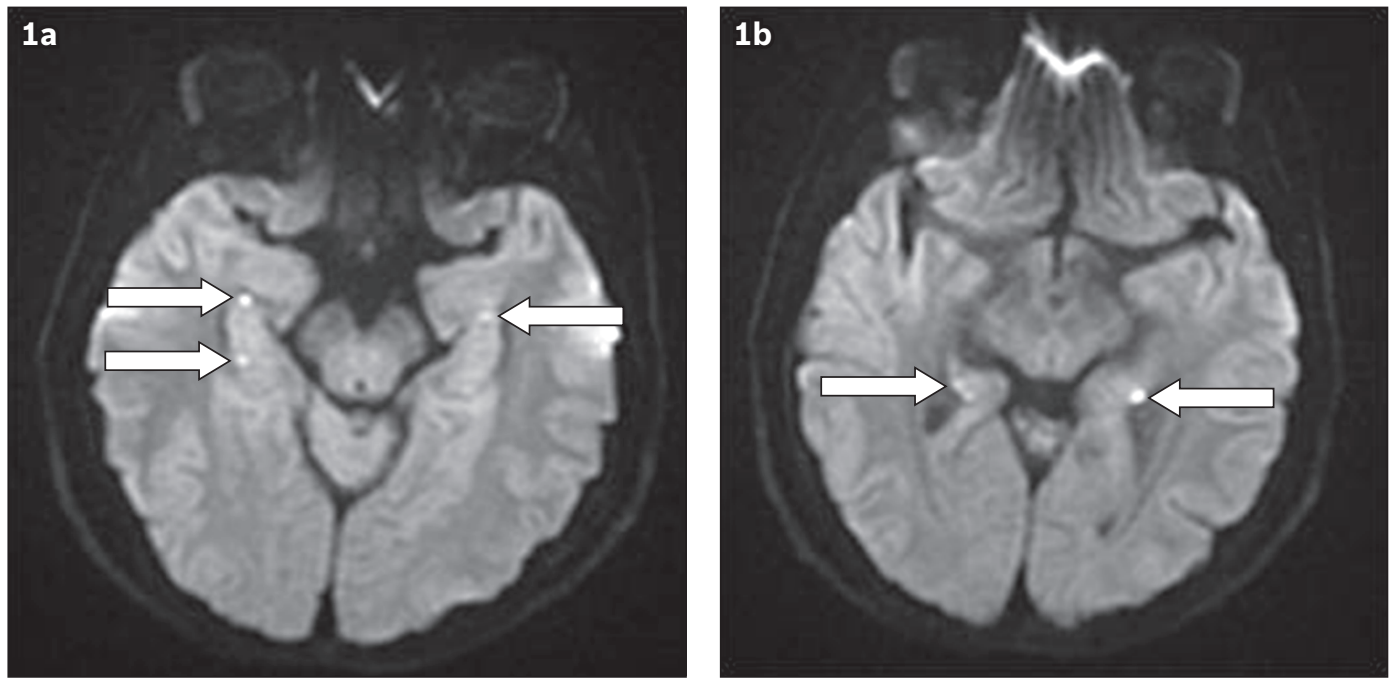

Fig. 1 DW imaging sequences at the level of (a) the cerebral peduncles and (b) lower mid-brain.

\section{CASE PRESENTATION}

A 60-year-old woman with no significant past medical history was travelling on a plane with her spouse when she suddenly developed acute onset of disorientation to time and space. According to her husband, she repeatedly asked where she was and complained of a minor headache. Throughout the episode of disorientation, the patient remained conscious and was cognisant of her identity. The patient's memories prior to boarding the plane were fully preserved. No focal abnormality was noted on neurological examination. Magnetic resonance (MR) imaging was performed (Fig. 1). What do the images show? What is the diagnosis?

${ }^{1}$ Department of Medical Imaging, Saskatoon Health Region, Saskatoon, Saskatchewan, Canada, ${ }^{2}$ Department of Radiology, Massachusetts General Hospital, Boston, Massachusetts, USA, ${ }^{3}$ Department of Diagnostic Radiology, Singapore General Hospital, Singapore

Correspondence: Dr Robert Chen, Senior Consultant Radiologist, Department of Diagnostic Radiology, Singapore General Hospital, Outram Road, Singapore 169608. robert.chen@sgh.com.sg 


\section{IMAGE INTERPRETATION}

Axial MR sequences through the level of the temporal lobes show several punctate $2-3 \mathrm{~mm}$ regions of diffusion-weighted (DW) imaging hyperintensity in the region of the lateral hippocampi (arrows in Figs. 1a \& b). The remainder of the brain is normal in appearance.

\section{DIAGNOSIS}

Transient global amnesia (TGA).

\section{CLINICAL COURSE}

The patient was admitted to a neurology ward for observation. Her disorientation gradually and spontaneously resolved over the course of 12 hours without intervention. The patient was subsequently discharged with outpatient follow-up. A short amnestic gap remained and she was unable to recall the events that occurred during the acute episode.

\section{DISCUSSION}

TGA manifests as a sudden and striking onset of anterograde amnesia that spontaneously resolves within 24 hours. Age is the only known risk factor, ${ }^{(1)}$ as it predominantly affects middle-aged people between the fifth and seventh decades of life. TGA has an incidence of up to 32 per 100,000 among those aged over 50, with an equal distribution between both genders and all racial groups. ${ }^{(2)}$

Although it was first documented in 1958, the pathophysiology behind TGA remains poorly understood. (3) A number of mechanisms have been proposed, including arterial ischaemia, venous congestion, migraine and epilepsy. ${ }^{(4)}$ However, convincing evidence to support these theories remains scant. With respect to the most popular theory of arterial ischaemia, a number of case-control studies have been unable to demonstrate a relationship between TGA and an increase in cerebrovascular risk factors (e.g. blood pressure and serum cholesterol) or other anatomical cardiac abnormalities. ${ }^{(5)}$ Furthermore, follow-up studies comparing TGA patients to transient ischaemic attack (TIA) patients have actually shown a significantly lower incidence of future stroke in the TGA group. ${ }^{(6)}$

Although the pathophysiology of TGA is unclear, the clinical presentation is quite characteristic and relatively specific. Currently, the diagnosis is made solely based on clinical criteria (Box 1).(7) Patients classically present with acute onset of disorientation to time and place with both anterograde and retrograde amnesia of variable duration. Anterograde amnesia will often present with repetitive questioning due to an inability to form new memories. However, long-term memory, executive function, language, visual-spatial orientation and all other higher order functions are preserved. ${ }^{(1)}$ In fact, TGA presents with no other focal neurological deficits, which makes the isolated amnesia all the more striking. Triggering events such as a valsalva manoeuvre, emotional distress, high altitude or postural changes have also been reported prior to symptom onset. ${ }^{(4)}$

Radiology can be particularly useful in confirming the presence of TGA in cases where the clinical criteria for TGA are not fully satisfied. The presence of solitary or multifocal 1-3 mm
Box 1. Transient global amnesia clinical criteria (derived from

Hodges and Warlow). ${ }^{(7)}$

- Attack witnessed by an observer

- Acute onset of anterograde amnesia

- No change of consciousness or loss of self-awareness

- Cognitive impairment limited to amnesia

- No recent history of head trauma or seizures

- Duration of symptoms of 1-24 hrs

- No neurological symptoms besides dizziness, vertigo or headache

punctate regions of restricted diffusion within the unilateral or bilateral hippocampi is very suggestive of TGA. ${ }^{(4,8,9)}$ According to Sedlaczek et al, based on a retrospective study of 31 cases, MR imaging may be positive in only $6 \%$ of cases in the first few hours after the initial attack. This percentage rises to $84 \%$ if scans are performed 48 hours after the initial symptoms. ${ }^{(8)}$ These detection rates were based on DW imaging slice thicknesses of $5 \mathrm{~mm}$ and b-values of $1000 \mathrm{~s} / \mathrm{mm}^{2}$ and $2000 \mathrm{~s} / \mathrm{mm}^{2}{ }^{\left({ }^{(8)}\right.}$ Further refinement of DW imaging parameters has improved the detection rate for TGA lesions to up to $88 \%$ using a slice thickness of $2 \mathrm{~mm}$, a b-value of $2000 \mathrm{~s} / \mathrm{mm}^{2}$ and repeat imaging at 72 hours. ${ }^{(10)}$ Therefore, if TGA is suspected, optimising DW imaging parameters and performing delayed imaging 48-72 hours after presentation will allow for the highest possible sensitivity.

MR signal abnormality affecting the hippocampi has a limited radiological differential diagnosis. A recent paper published by Förster et $\mathrm{al}^{\left({ }^{(9)}\right.}$ described how to differentiate patterns of diffusion restriction on DW imaging sequences from the more common processes affecting the hippocampus, such as infarction, TGA, seizure and limbic encephalitis. For example, the classic imaging appearance of TGA is of discrete, punctate 1-3 mm DW imaging lesions that are usually located towards the lateral aspect of the hippocampus (Fig. 2a). In contrast, a posterior cerebral artery (PCA) infarction affecting the hippocampus is typically larger than 1-3 $\mathrm{mm}$ in size and often has patchy DW images involving other portions of the PCA distribution such as the occipital lobe, thalamus and corpus callosum (Fig. 2b). In the acute phase of seizure, the majority of patients have normal MR imaging findings. However, in those with detectable brain signal abnormality on MR imaging, there may be diffuse signal abnormality and swelling in the hippocampi, and additional signal abnormality can sometimes be seen in the pulvinar and/or cortex (Figs. 2c-e). Finally, limbic encephalitis affects a larger portion of the mesial temporal lobe than just the hippocampus, and can affect all the limbic structures as well, such as the parahippocampal gyrus, insula and cingulate gyrus $^{(11)}$ (Figs. 2f-h). Limbic encephalitis is frequently bilateral.

The clinical differential diagnosis for the presentation of isolated short-term retrograde amnesia with the inability to form new memories includes TGA, vascular entities such as TIA/stroke, transient epileptic amnesia and, less commonly, a toxic or metabolic disturbance. A full neurological examination and history can usually confirm the diagnosis in most cases. Clinically, epileptic amnesia will typically be of a shorter duration, and may present with automatisms such as lip smacking or abnormal electroencephalography (EEG) findings. ${ }^{(12)}$ Toxic or 

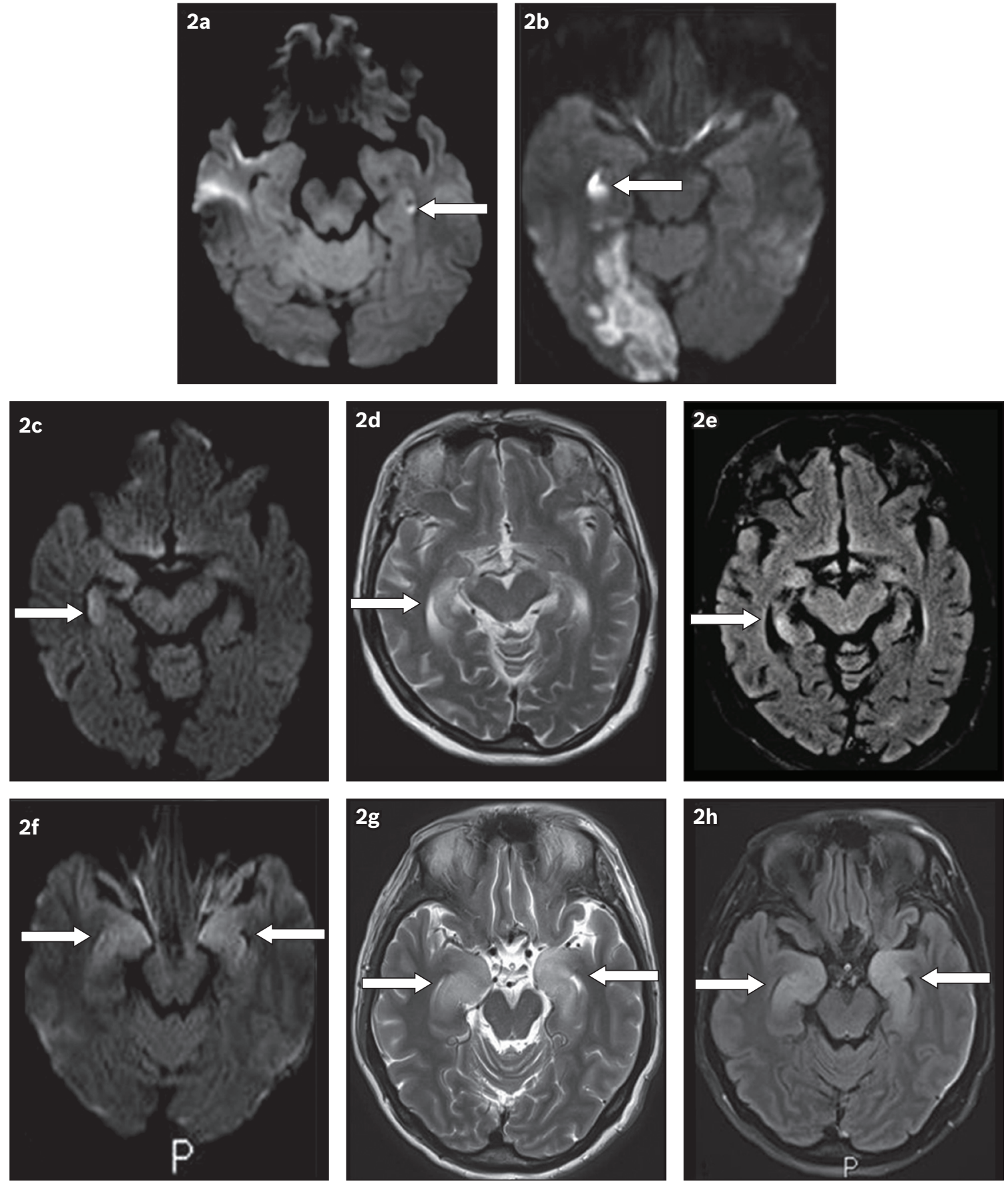

Fig. 2 Comparison of DW patterns of hyperintensity within the hippocampus. (a) MR image of transient global amnesia shows a punctate focus (arrow) of signal abnormality in the left hippocampus. (b) MR image of posterior cerebral artery (PCA) infarct shows a larger region of signal abnormality (arrow) in the right hippocampus with associated patchy DW signal in the right occipital lobe (PCA distribution). (c-e) MR images of seizure-related change, with corresponding (d) T2-W and (e) FLAIR images, show increased signal abnormality (arrow in 2c) and swelling in the right hippocampus (arrow in 2d) that reversed upon subsequent imaging (arrow in 2e). ( $f-h$ ) MR images of limbic encephalitis with corresponding (g) T2-W and (h) FLAIR images which show signal abnormality and swelling in both hippocampi (arrows).

metabolic states such as hypoglycaemia usually have features that are consistent with global cognitive dysfunction, such as a delirious state.

In the case of a vascular process such as TIA, an embolism is more likely to shower across multiple small arterioles ${ }^{(9)}$ to produce a more diffuse clinical picture involving more than just retrograde and anterograde amnesia. Nevertheless, if there is still diagnostic uncertainty after a full neurological exam, blood tests for electrolyte balance, glucose levels and complete blood count, toxicology screen, EEG and neuroimaging with MR imaging are recommended.
Once diagnosed, the prognosis for TGA is largely favourable. Episodes commonly resolve within six hours, but may last up to 24 hours before resolving spontaneously. Patients can be left with a permanent amnestic gap corresponding to the episode of anterograde amnesia. Besides a brief amnestic gap, patients will have no long-term neurological deficits, and the risk of reoccurrence is relatively low, in approximately $5 \%$ of patients. ${ }^{(13)}$ Management should include watchful waiting, outpatient follow-up and repeat MR imaging if clinically indicated. (1) 
ABSTRACT A 60-year-old woman was travelling on a plane with her spouse when she suddenly developed acute-onset disorientation to time and space. According to her husband, she repetitively questioned her whereabouts and complained of a minor headache. Upon landing, she was immediately brought to the emergency room and subsequent magnetic resonance imaging was performed, which showed multiple punctate 2-3 mm regions of diffusion-weighted imaging hyperintensity in the medial aspects of both temporal lobes. The conglomeration of clinical history and radiological findings was most suggestive of transient global amnesia. This article discussed the background, proposed mechanisms, diagnosis, radiological characteristics and management of transient global amnesia.

Keywords: diffusion weighted imaging, transient global amnesia

\section{REFERENCES}

1. Kremen S. Transient global amnesia. In: UpToDate [online]. Available at: http:// www.uptodate.com/contents/transient-global-amnesia. Accessed February 23, 2017.
2. Koski KJ, Marttila RJ. Transient global amnesia: incidence in an urban population. Acta Neurol Scand 1990; 81:358-60.

3. Fisher CM, Adams RD. Transient global amnesia. Acta Neurol Scand Suppl 1964; 40 Suppl 9:1-83.

4. Sander K, Sander D. New insights into transient global amnesia: recent imaging and clinical findings. Lancet Neurol 2005; 4:437-44.

5. Enzinger C, Thimary F, Kapeller $\mathrm{P}$, et al. Transient global amnesia: diffusionweighted imaging lesions and cerebrovascular disease. Stroke 2008; 39:2219-25.

6. Mangla A, Navi BB, Layton K, Kamel H. Transient global amnesia and the risk of ischemic stroke. Stroke 2014; 45:389-93.

7. Hodges JR, Warlow CP. Syndromes of transient amnesia: towards a classification. A study of 153 cases. J Neurol Neurosurg Psychiatry 1990; 53:834-43.

8. Sedlaczek O, Hirsch JG, Grips E, et al. Detection of delayed focal MR changes in the lateral hippocampus in transient global amnesia. Neurology 2004; 62:2165-70.

9. Förster A, Griebe M, Gass A, et al. Diffusion-weighted imaging for the differential diagnosis of disorders affecting the hippocampus. Cerebrovasc Dis 2012; 33:104-15.

10. Kim J, Kwon Y, Yang Y, et al. Clinical experience of modified diffusion-weighted imaging protocol for lesion detection in transient global amnesia: an 8-year large-scale clinical study. J Neuroimaging 2014; 24:331-7.

11. Pearce MC, Chen R. Non-paraneoplastic limbic encephalitis with voltage gated potassium channel (VGKC) antibodies. Am Coll Radiol Case Point Series 2014.

12. Bartsch T, Butler C. Transient amnesic syndromes. Nat Rev Neurol 2013; 9:86-97.

13. Quinette P, Guillery-Girard B, Dayan J, et al. What does transient global amnesia really mean? Review of the literature and thorough study of 142 cases. Brain 2006; 129(Pt 7):1640-58. 


\section{SINGAPORE MEDICAL COUNCIL CATEGORY 3B CME PROGRAMME} (Code SMJ 201807B)

Question 1. Regarding transient global amnesia (TGA):

(a) TGA manifests with sudden-onset amnesia and automatisms, which both spontaneously resolve over 24 hours.

(b) The condition has an equal incidence in men and women.

(c) It affects patients in their fifth to seventh decade of life.

(d) TGA is associated with transient visual impairment and seizure activity.

Question 2. Regarding patients presenting with memory loss:

(a) The differential should always include transient epileptic amnesia, stroke and transient ischaemic attack (TIA).

(b) Abnormal electroencephalography findings are not associated with TGA.

(c) TIA rarely presents with isolated retrograde and anterograde amnesia.

(d) Executive and language dysfunction associated with memory loss are not likely in TGA.

Question 3. Regarding the clinical and radiologic workup of suspected TGA patients:

(a) Computed tomography is highly sensitive to the changes of TGA.

(b) Magnetic resonance (MR) imaging cannot distinguish between TIA and TGA.

(c) Laboratory workup may include obtaining a complete blood count, glucose level and serum electrolyte balance.

(d) MR imaging is most sensitive for TGA findings when performed 48 hours after symptom onset.

Question 4. Regarding the evaluation of TGA with MR imaging:

(a) Atrophy of the hippocampus is a common chronic change associated with TGA.

(b) Patchy diffusion-weighted (DW) imaging hyperintensity may involve the parahippocampal gyrus.

(c) Small punctate DW imaging hyperintensities can be seen in the lateral hippocampi.

(d) Signal abnormality may be seen in the pulvinar and thalamus.

Question 5. Regarding the management and prognosis of TGA:

(a) Patients can be left with a permanent amnestic gap.

(b) Recurrence risk can be as high as 35\%.

(c) Routine follow-up MR imaging is recommended for most cases of TGA.

(d) Surgical management is not indicated for this condition.

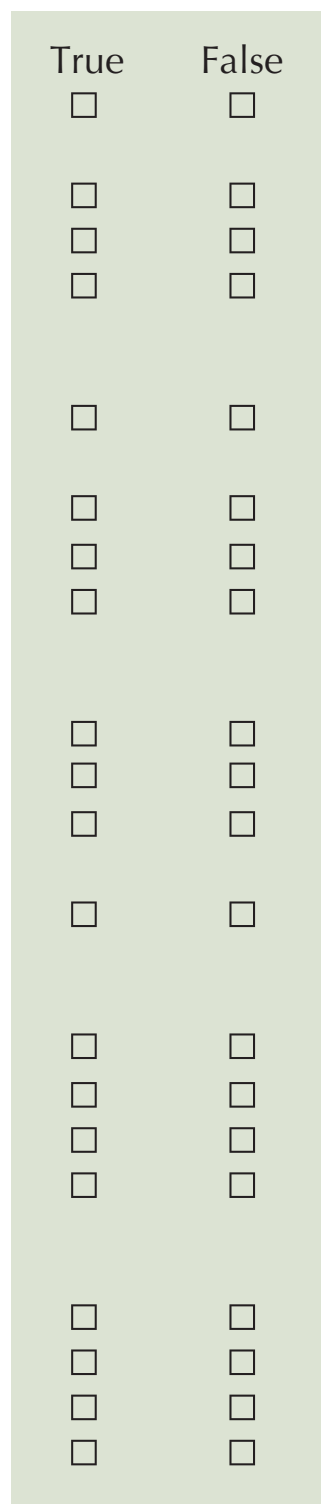

Doctor's particulars:

Name in full:

MCR no.:

Specialty:

Email:

\section{SUBMISSION INSTRUCTIONS:}

Visit the SMJ website: http://www.smj.org.sg/current-issue and select the appropriate quiz. You will be redirected to the SMA login page.

For SMA member: (1) Log in with your username and password (if you do not know your password, please click on 'Forgot your password?'). (2) Select your answers for each quiz and click 'Submit'.

For non-SMA member: (1) Create an SMJ CME account, or log in with your SMJ CME username and password (for returning users). (2) Make payment of SGD 21.40 (inclusive of $7 \%$ GST) via PayPal to access this month's quizzes. (3) Select your answers for each quiz and click 'Submit'.

\section{RESULTS:}

(1) Answers will be published online in the SMJ September 2018 issue. (2) The MCR numbers of successful candidates will be posted online at the SMJ website by 5 September 2018. (3) Passing mark is $60 \%$. No mark will be deducted for incorrect answers. (4) The SMJ editorial office will submit the list of successful candidates to the Singapore Medical Council. (5) One CME point is awarded for successful candidates. (6) SMC credits CME points according to the month of publication of the CME article (i.e. points awarded for a quiz published in the December 2017 issue will be credited for the month of December 2017, even if the deadline is in January 2018).

Deadline for submission: (July 2018 SMJ 3B CME programme): 12 noon, 29 August 2018. 